Article

\title{
Biaxial Yield Surface Investigation of Polymer-Matrix Composites
}

\section{Junjie Ye ${ }^{1} *$, Yuanying Qiu ${ }^{1}$, Zhi Zhai ${ }^{2}$ and Zhengjia $\mathrm{He}^{2}$}

1 Key Laboratory of Ministry of Education for Electronic Equipment Structure Design, Xidian University, Xi’an 710071, China; E-Mail: yyqiu@ mail.xidian.edu.cn

2 State Key Laboratory for Manufacturing Systems Engineering, Xi'an Jiaotong University, Xi'an 710049, China; E-Mails: elmar@stu.xjtu.edu.cn (Z.Z.); hzj@mail.xjtu.edu.cn (Z.H.)

* Author to whom correspondence should be addressed; E-Mail: ronkey6000@ sina.com; Tel./Fax: +86-29-8820-4639.

Received: 1 March 2013; in revised form: 18 March 2013 / Accepted: 18 March 2013 /

Published: 25 March 2013

\begin{abstract}
This article presents a numerical technique for computing the biaxial yield surface of polymer-matrix composites with a given microstructure. Generalized Method of Cells in combination with an Improved Bodner-Partom Viscoplastic model is used to compute the inelastic deformation. The validation of presented model is proved by a fiber Bragg gratings (FBGs) strain test system through uniaxial testing under two different strain rate conditions. On this basis, the manufacturing process thermal residual stress and strain rate effect on the biaxial yield surface of composites are considered. The results show that the effect of thermal residual stress on the biaxial yield response is closely dependent on loading conditions. Moreover, biaxial yield strength tends to increase with the increasing strain rate.
\end{abstract}

Keywords: biaxial yield surface; thermal residual stress; fiber off-axis angle; strain rate

\section{Introduction}

Due to their remarkable mechanical characteristics and wide range of potential applications, composites have attracted extensive attention of researchers. Composites present evident plastic behavior, which is primarily characterized by yielding and rate sensitivity in service. Because of the complexity of composite materials, experimental methods require considerable financial and human resources. Furthermore, compared with uniaxial loading conditions, it is difficult to acquire yield 
strength of composites through macroscopic experimental methods under complex stress conditions. Therefore, more and more investigators rely to theoretical research on biaxial yield responses of composites.

Two main methods, namely the analytical micromechanical method and the finite element method, have been used to study yield the behaviors of composites under complex stress conditions. Azizi et al. [1] utilized a finite element method to study the size-effects on initial yield surfaces, and subsequent yield surfaces for reinforced composites under axial-torsion loading conditions. Tang and $\mathrm{Yu}$ [2] adopted a finite element method to predict the initial yielding surface of heterogeneous materials with realistic microstructures. Moshtaghin et al. [3] constructed a micromechanical method to investigate the effects of surface residual stress as well as surface elasticity on the overall yield strength of nanoporous metal matrices containing aligned cylindrical nanovoids. Acton and Graham [4,5] used a moving window Generalized Method of Cells to approximate a yield surface. Moreover, in order to determine the accuracy of the models, each result was compared with an analytical study. Through application of the Mean-field Homogenization method, Selmi et al. [6] predicted the biaxial yield behavior, hardening and plastic flow of misaligned short fiber-reinforced composites. For laminated metal matrix composites, Radi and Abdul [7] described the evolution of the yield surface using a recent developed self-consistent model with small strains assumption. Lissenden $[8,9]$ using a proof strain criterion for the permanent strain that relies on cyclic, proportional to probe the loci of the yield surface. Furthermore, initial and subsequent yield surfaces of highly anisotropic materials were studied by experimental methods. However, few studies concerning the thermal residual stress and strain rate influence on the yield surface of composites with different fiber off-axis angles have been reported.

In addition, it should be noted that traditional strain gauges can hardly capture dynamic strain changes exactly under high-rate loading conditions due to the sensitivity to electromagnetic interference and low speed response. In this paper, repeatability and sensitivity of FBGs sensor are validated by a cantilever system. Meanwhile, the prediction results under uniaxial tensile conditions are validated by experimental data of a FBGs strain test system. On this basis, the effects of thermal residual stress and strain rate on the yield surfaces of composites with different fiber off-axis angles are investigated.

\section{Micro-Mechanical Models of Fiber-Reinforced Composites}

\subsection{Representative Volume Element}

In the most micro-mechanical models, it is supposed that inclusions or fibers present periodic configuration in the composites, as shown in Figure 1. Through choosing a proper unit, a micro-mechanical constitutive model of the composites can be established. On this basis, macro-mechanical behaviors can be acquired from the homogenization theory. 
Figure 1. Fiber-reinforced composites with periodic array.

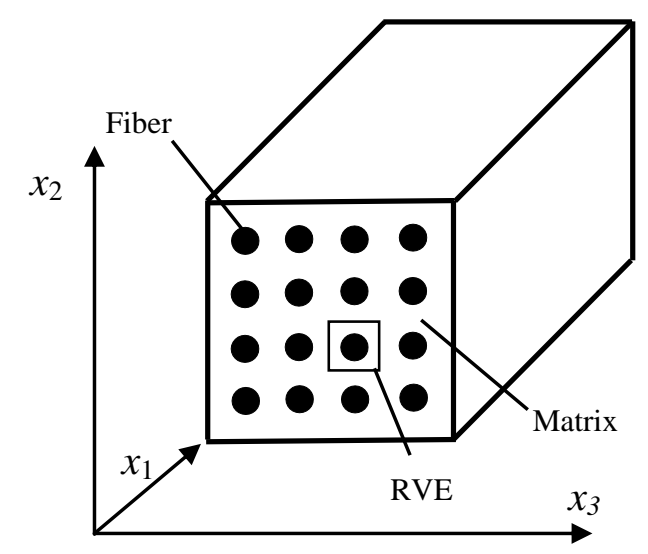

\subsection{Generalized Method of Cells}

Generalized Method of Cells (GMC), one of the most important micromechanical models, has been used in predicting effective elastic constants, mechanical properties of composites [10-12]. For fiber-reinforced composites, the representative volume element (RVE) is extracted from the cross section which is perpendicular to the fiber direction. The RVE is divided into $N_{\beta} \times N_{\gamma}$ sub-cells as shown in Figure 2. In the figure, $h$ and $l$ indicate the length of the RVE in the $y_{2}$ and $y_{3}$ directions, respectively. $\beta$ and $\gamma$ indicate the number of the sub-cells in the $y_{2}$ and $y_{3}$ directions, respectively. The constitutive equation of sub-cells is given by:

$$
\bar{\sigma}^{(\beta \gamma)}=C^{(\beta \gamma)}\left(\bar{\varepsilon}^{(\beta \gamma)}-\bar{\varepsilon}^{P(\beta \gamma)}-\alpha^{(\beta \gamma)} \Delta T\right)
$$

where $\bar{\sigma}^{(\beta \gamma)}$ is the average stress of the sub-cells. $C^{(\beta \gamma)}$ is the stiffness matrix of the sub-cells. $\bar{\varepsilon}^{(\beta \gamma)}$ and $\bar{\varepsilon}^{P(\beta \gamma)}$ indicate the average strain and plastic strain of the sub-cells. $\alpha^{(\beta \gamma)}$ and $\Delta T$ indicate thermal expansion coefficient of the sub-cells and temperature change.

Figure 2. Discretization of the RVE.

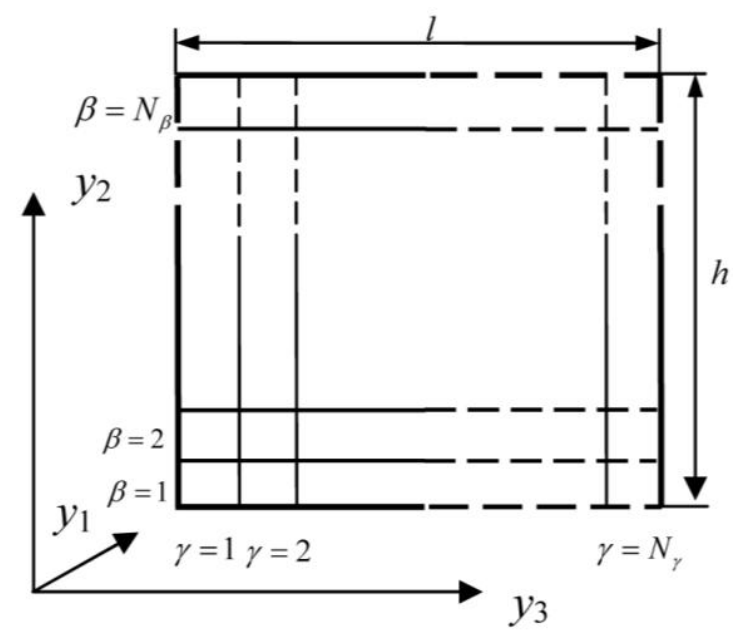

According to the homogenization theory, the relationship between macroscopic average stress $\bar{\sigma}$ and sub-cell average stress $\bar{\sigma}^{(\beta \gamma)}$ can be expressed as: 


$$
\begin{aligned}
\bar{\sigma} & =\frac{1}{h l} \sum_{h=1}^{N_{\beta}} \sum_{\gamma=1}^{N_{\gamma}} \bar{\sigma}^{(\beta \gamma)} \\
& =C^{*}\left(\bar{\varepsilon}-\bar{\varepsilon}^{p}-\alpha \Delta T\right)
\end{aligned}
$$

where $C^{*}$ is macroscopic stiffness of composites, $\bar{\varepsilon}$ and $\bar{\varepsilon}^{P}$ indicate the macroscopic average strain and plastic strain, respectively. $\alpha$ is macroscopic thermal expansion coefficient.

In order to satisfy displacement continuity conditions between adjacent sub-cells and axial deformation constraint conditions, the relationship between sub-cell average strain and macro strain can be expressed as follows:

$$
A_{G} \bar{\varepsilon}_{s}=J \bar{\varepsilon}
$$

where $A_{G}$ contains geometric dimension of the sub-cells. $J$ contains the geometric dimension of the RVE. $\bar{\varepsilon}_{S}$ indicates strain vector of the sub-cells. $\bar{\varepsilon}$ is the component of macro-strain.

According to the stress continuity conditions between sub-cells and the constitutive equation of the sub-cells, the relationship among average plastic strain components $\bar{\varepsilon}_{S}^{P}$, average thermal strain components $\bar{\varepsilon}_{S}^{T}$, and average strain components $\bar{\varepsilon}_{S}$ can be established as follows:

$$
A_{M}\left(\bar{\varepsilon}_{s}-\bar{\varepsilon}_{s}^{p}-\bar{\varepsilon}_{s}^{T}\right)=0
$$

where $A_{M}$ contains the stiffness matrix of the sub-cells.

Combining Equations (3) and (4), the sub-cells strain vector $\bar{\varepsilon}_{S}$ can be acquired. Furthermore, average sub-cell strains $\bar{\varepsilon}^{(\beta \gamma)}$ can be expressed as:

$$
\bar{\varepsilon}^{(\beta \gamma)}=A^{(\beta \gamma)} \bar{\varepsilon}+D^{(\beta \gamma)}\left(\bar{\varepsilon}_{S}^{P}+\bar{\varepsilon}_{S}^{T}\right)
$$

where concentrations matrices $A^{(\beta \gamma)}$ refers to stiffness matrix as well as geometric dimensions of the sub-cells. $D^{(\beta \gamma)}$ refers to the elastic constants of the sub-cell materials.

Substituting Equation (5) into Equation (1), sub-cell average stress $\bar{\sigma}^{(\beta \gamma)}$ can be acquired. On this basis, substituting $\bar{\sigma}^{(\beta \gamma)}$ into Equation (2), macroscopic stress $\bar{\sigma}$ can be written as:

$$
\bar{\sigma}=\frac{1}{h l} \sum_{\beta=1}^{N_{\beta}} \sum_{\gamma=1}^{N_{\gamma}} h_{\beta} l_{\gamma} C^{(\beta \gamma)}\left[A^{(\beta \gamma)} \bar{\varepsilon}+D^{(\beta \gamma)}\left(\bar{\varepsilon}_{S}^{P}+\bar{\varepsilon}_{S}^{T}\right)-\left(\bar{\varepsilon}_{S}^{P(\beta \gamma)}+\bar{\varepsilon}_{S}^{T(\beta \gamma)}\right)\right]
$$

Through comparing Equation (6) with Equation (2), macroscopic stiffness matrix $C^{*}$ can be expressed as follows:

$$
C^{*}=\frac{1}{h l} \sum_{\beta=1}^{N_{\beta}} \sum_{\gamma=1}^{N_{\gamma}} h_{\beta} l_{\gamma} C^{(\beta \gamma)} A^{(\beta \gamma)}
$$

\section{Experimental Verification}

\subsection{Experimental Research on a FBGs Sensor}

Due to their small dimensions and accurate measurements, as well as resistance to corrosion and electromagnetism, FBGs sensors have been used in cardiac ablation [13], structural health monitoring [14,15], as well as the biomechanics and rehabilitation fields [16]. The structure of a FBGs sensor can be seen in Figure 3. It is composed of an optical fiber, grating and fiber core. The 
fabrication process of FBGs sensor is due to the photosensitivity property of the doped silica glass fiber core. A permanent grating can be inscribed into the photosensitive fiber core when exposed to ultraviolet light and is usually obtained by means of the two-beam interference technique or phase mask method. The principle of the FBGs sensor is to measure the changes of center wavelengths of reflective light from a Bragg grating. With the variation of strain, the center wavelengths of the reflection light will be corresponding changed. The Bragg wavelength can be expressed as follows [17]:

$$
\lambda_{B}=2 n_{e f f} \Lambda
$$

where $n_{\text {eff }}$ is the effective reflective index of the fiber core, $\Lambda$ is the grating periodic spacing, $\lambda_{B}$ is the wavelength of reflected light. It can be found from Equation (8) that the Bragg wavelength will shift with the parameter of $n_{\text {eff }}$ and $\Lambda$. Disregarding the thermal influence, the periodic spacing and effective reflective index will change when the mechanical deformation is posed on the grating area. The relationship between Bragg wavelength shift and the change of strain $(\Delta \varepsilon)$ can be expressed as:

$$
\Delta \lambda_{B}=\alpha \Delta \varepsilon
$$

where:

$$
\alpha=\lambda_{B}\left(1-p_{e}\right)
$$

and $p_{e}$ is an effective strain-optic constant.

Figure 3. The structure of FBGs sensor.

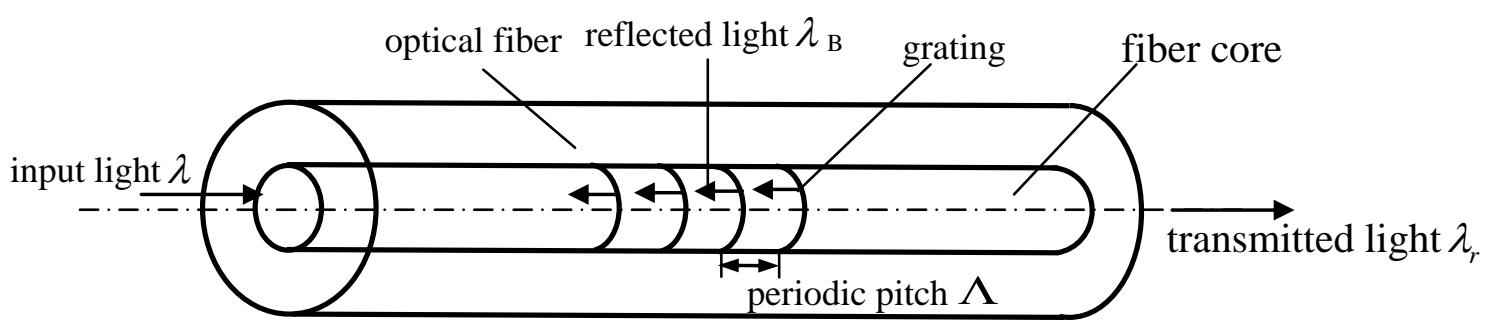

In acquiring strain signals, the next two methods are always used [18]: directly pasted on surface or embedded into structures. In this paper, the former method is used to test the strains of composites. Before making use of the FBGs sensor, repeatability and sensitivity experiments are performed. In the experiment, a SM130-700 fiber grating demodulator, which is produced by Micron Optical International Corporation, is used to measure optical signal. The parameter of SM130-700 is as follows: Wavelength scanning range is $1,510-1,590 \mathrm{~nm}$. Resolution is $1 \mathrm{pm}$. Scanning frequency is $1,000 \mathrm{~Hz}$.

\subsubsection{Repeatability Experiment}

In order to validate the strain test of FBGs sensor, a cantilever beam structure is used as shown in Figure 4. The corresponding parameters are as follows: the width and height of the cantilever beam are $12 \mathrm{~mm}$ and $20 \mathrm{~mm}$, respectively. Elastic modulus is $206 \mathrm{Gpa}$. The parameter $a=424 \mathrm{~mm}$ indicates the distance between central position of FBGs sensor and the fixed end of the cantilever beam. The parameter $L=469 \mathrm{~mm}$ indicates the distance between the loading position and the fixed end of 
cantilever beam. Loading sequence is as follows: $19.6 \mathrm{~N}, 39.2 \mathrm{~N}, 49.0 \mathrm{~N}, 58.8 \mathrm{~N}, 68.6 \mathrm{~N}$. The relationship between measuring wavelength $A_{i}$ of FBGs sensor and measuring strain $\varepsilon$ can be written as follows [19]:

$$
\varepsilon=\left(A_{i}-A_{0}\right) * 1000 / 1.2
$$

where $A_{0}$ indicate initial wavelength. The parameter 1,000 converts the Bragg wavelength shift from $\mathrm{nm}$ to $\mathrm{pm}$. The parameter 1.2 indicates the strain sensitivity of the FBGs sensor, which is provided by Micron Optical International Corporation.

Figure 4. Test principle diagram.

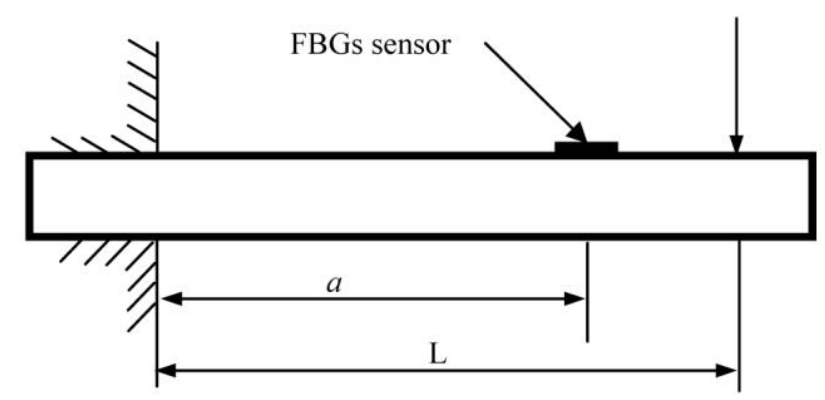

Measurement data are linear fitted by the least-squares method. Figure 5(a,b) shows the experimental results under loading and unloading conditions, respectively. It can be seen that measured results of the FBGs sensor show high reproducibility under loading and unloading conditions. Figure 6 shows the wavelength absolute error of the FBGs sensor between the theoretical wavelength and average wavelength of the test results. It can be seen from the figure that absolute error is less than $3 \mathrm{pm}$.

Figure 5. Repeatability experiment of FBGs sensor: (a) Loading conditions. (b) Unloading conditions.

(a)

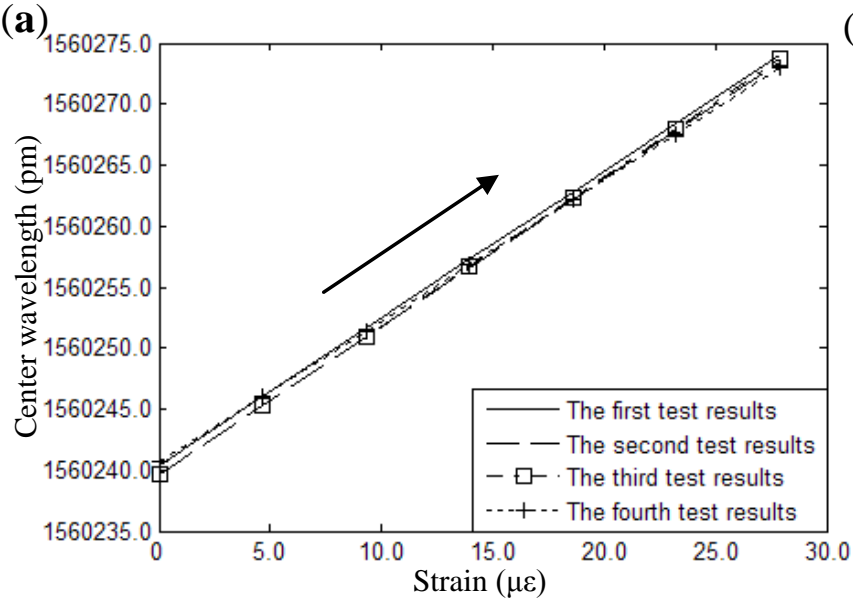

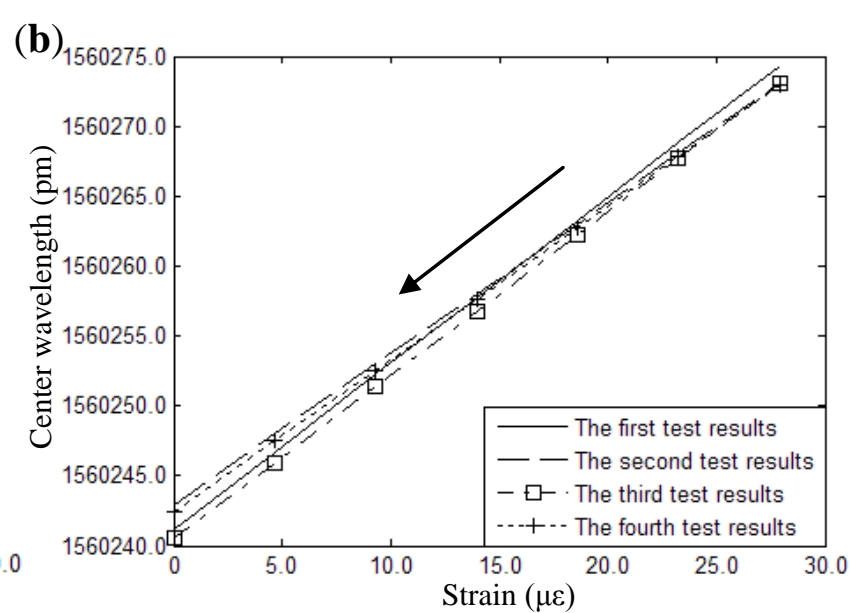


Figure 6. Wavelength absolute error of the FBGs sensor under loading and unloading conditions.

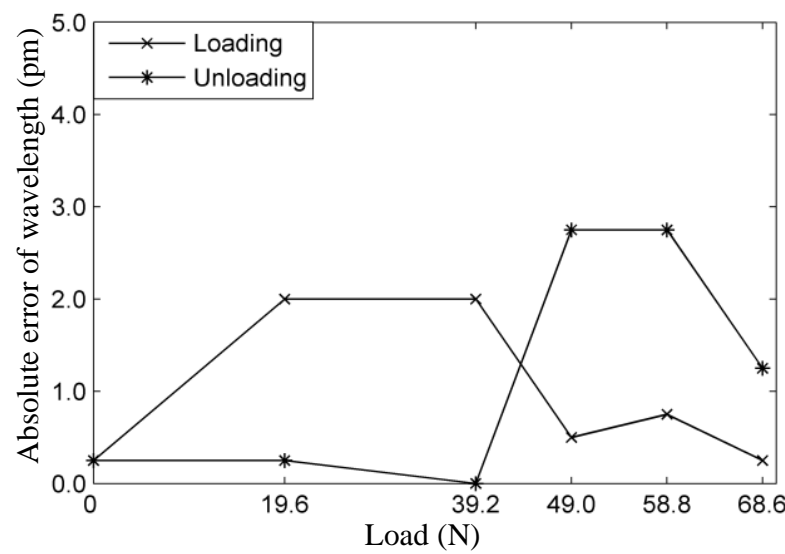

\subsubsection{Sensitivity Experiment}

The average wavelengths of four repeated tests are used to fit the strain sensitivity coefficients by the least-squares method under loading and unloading conditions. The sensitivity experimental results and corresponding error analysis can be seen in Figure 7 and Table 1, respectively. Moreover, the theoretical result of the strain sensitivity coefficient (1.200), which can be acquired from Equation (11), are also shown in the figures. From the Table 1, it can be seen that the strain sensitivity coefficients of the FBGs sensor under loading and unloading conditions are 1.194 and 1.158, respectively. Comparing with the theoretical results, the relative errors of the experimental results are $0.50 \%$ and $3.50 \%$, respectively. In addition, correlation coefficients of the theoretical curve and the fitted curve under conditions of loading and unloading are 0.9959 and 0.9948 , respectively. Based on the studies mentioned above, it can be seen that the FBGs sensor directly pasted on the structure presents high selectivity in strain tests.

Figure 7. Sensitivity experiment of the FBGs sensor: (a) Loading conditions. (b) Unloading conditions.
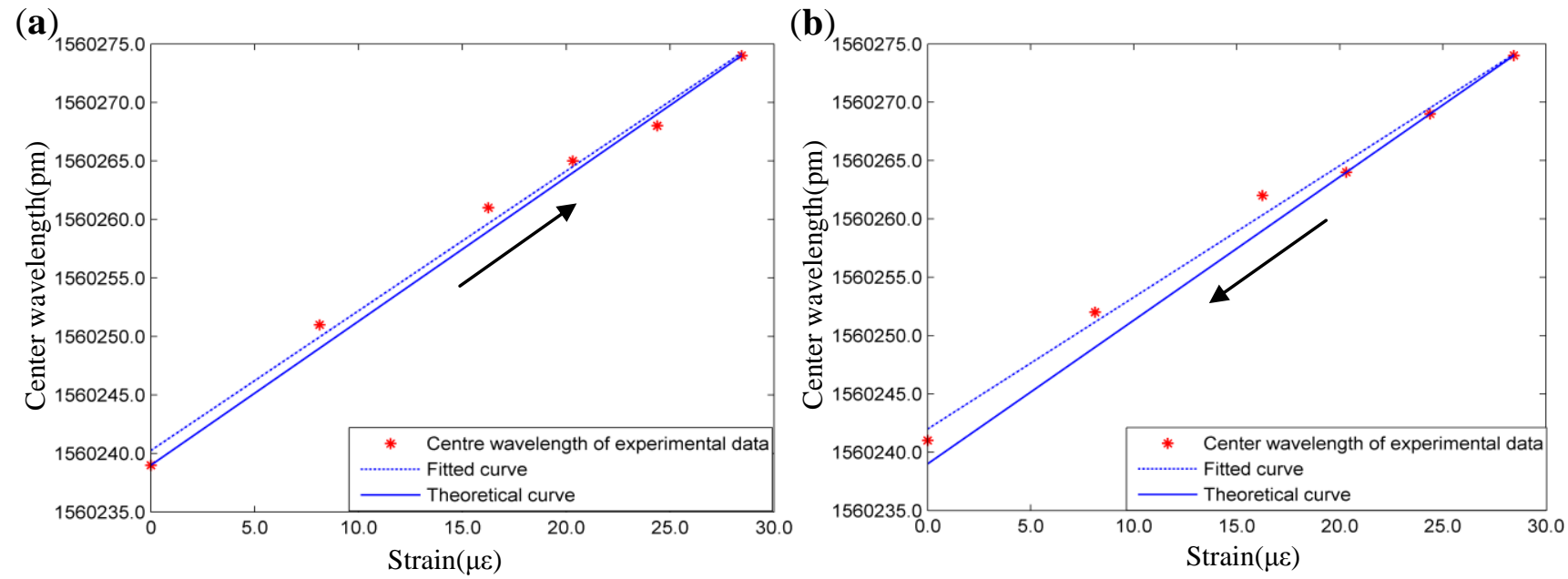
Table 1. Sensitivity and initial wavelength error analysis of FBGs sensor.

\begin{tabular}{ccccccc}
\hline $\begin{array}{c}\text { Loading } \\
\text { condition }\end{array}$ & $\begin{array}{c}\text { Theoretical } \\
\text { value } \boldsymbol{\lambda}_{\mathbf{0}} / \mathbf{n m}\end{array}$ & $\begin{array}{c}\text { Measured } \\
\text { value } \boldsymbol{\lambda}_{\mathbf{0}} / \mathbf{n m}\end{array}$ & $\begin{array}{c}\text { Absolute } \\
\text { error } \boldsymbol{\lambda}_{\mathbf{0}} / \mathbf{n m}\end{array}$ & $\begin{array}{c}\text { Sensitivity } \\
/(\mathbf{p m} / \boldsymbol{\mu} \boldsymbol{\varepsilon})\end{array}$ & $\begin{array}{c}\text { Sensitivity } \\
\text { relative error }\end{array}$ & $\begin{array}{c}\text { Related } \\
\text { coefficient }\end{array}$ \\
\hline $\begin{array}{c}\text { loading } \\
\text { unloading }\end{array}$ & 1560.2390 & 1560.2403 & 0.0013 & 1.194 & $0.50 \%$ & 0.9959 \\
\hline
\end{tabular}

\subsection{Experimental Research on Micromechanical Model}

In order to describe the nonlinear behaviors of polymer matrix composites, an Improved BodnerPartom (IBP) model is incorporated into the GMC model. Supposing that the fiber is linearly elastic, the polymer matrix is viscoplastic. The flow law for the viscoplastic strain rate components of the IBP model is formulated as follows [20]:

$$
\dot{\varepsilon}_{i j}^{I}=2 D_{0} \exp \left[-\frac{1}{2}\left(\frac{Z}{\sigma_{e}}\right)^{2 n}\right]\left(\frac{S_{i j}}{2 \sqrt{J_{2}}}+\alpha \delta_{i j}\right)
$$

where

$$
\begin{gathered}
J_{2}=\frac{1}{2} s_{i j} s_{i j} \quad \dot{Z}=q\left(Z_{1}-Z\right) \dot{e}_{e}^{I} \quad \dot{\alpha}=q\left(\alpha_{1}-\alpha\right) \dot{e}_{e}^{I} \\
\dot{e}_{e}^{I}=\sqrt{\frac{2}{3} \dot{e}_{i j}^{I} \dot{e}_{i j}^{I}} \dot{e}_{i j}^{I}=\dot{\varepsilon}_{i j}^{I}-\dot{\varepsilon}_{m}^{I} \delta_{i j}
\end{gathered}
$$

In the above, the overhead dot of the variables indicates the differentiation with respect to time $t$. Material parameters $n, Z_{0}, Z_{1}, \alpha_{0}, \alpha_{1}$ and $q$, which can be acquired by axial tension and pure shear experimental tests, are referred to as hardening characteristics. Six material parameters of the polymer matrix mentioned above can be determined through tensile and shear tests. According to the ASTM D3039 test, the dimension of the polymer specimen is $250 \times 25 \times 3 \mathrm{~mm}$. According to the ASTM D5379, the length and width of polymer specimen is $76.2 \times 19.1 \mathrm{~mm}$, while the thickness of the polymer specimen can be determined as required. In the experiment, uniaxial tension tests with strain rate $10^{-5} / \mathrm{s}$ and pure shear tests with strain rates $10^{-5} / \mathrm{s}$ and $10^{-1} / \mathrm{s}$ are used to determine the six material parameters. The details can be seen in the reference [21].

Figure $8(a, b)$ shows the picture and schematic of experimental system. The test system is made up of a Fiber Bragg Gratings (FBGs) sensor, composites specimen, material testing system and FBGs demodulation devices. Aluminum alloy tabs of $1 \mathrm{~mm}$ thickness were attached to the two ends of the specimen. The elastic modulus, Poisson's ration as well as viscoplastic parameters can be seen in Table 2. To validate the presented model, the uniaxial tensile mechanical responses of $15^{\circ}, 30^{\circ}, 45^{\circ}$ fiber-reinforced composites with 0.24 fiber volume fraction were measured under two different strain rate $(0.00001 / \mathrm{s}, 0.01 / \mathrm{s})$ conditions. Theoretical results and experimental data are shown in Figure 9 . It can be seen that theoretical prediction in different strain rate conditions shows excellent agreement with the experimental results. Comparing Figure 9(a) with Figure 9(b), it can be easily found that increasing strain rate will increase the yield strength of composites under uniaxial tension. For instance, compared with the strain rate $0.00001 / \mathrm{s}$, polymer-matrix composites provide a stress at the $2.5 \%$ strain that is approximately $20 \%$ higher than the stress of the composites with a strain rate of $0.01 / \mathrm{s}$ under $15^{\circ}$ fiber off-axis angle conditions. 
Table 2. Material parameters of the glass fiber and polymer matrix.

\begin{tabular}{ccccccccccc}
\hline Material & $\begin{array}{c}\text { Elastic modulus } \\
\boldsymbol{E} / \mathbf{G P a}\end{array}$ & $\begin{array}{c}\text { Poisson's } \\
\text { ratio } \boldsymbol{v}\end{array}$ & $\boldsymbol{n}$ & $\alpha_{1}$ & $\alpha_{0}$ & $\begin{array}{c}Z_{0} \\
\text { /Gpa }\end{array}$ & $\begin{array}{c}Z_{1} \\
\text { /Gpa }\end{array}$ & $\begin{array}{c}D_{0}^{-1} \\
/ \mathbf{s}\end{array}$ & $\boldsymbol{q}$ & $\alpha$ \\
\hline glass fiber & 71.42 & 0.2 & - & - & - & - & - & - & - & $5 \times 10^{-6}$ \\
Polymer & 3.3 & 0.22 & 0.63 & 0.104 & 0.184 & 0.391 & 0.803 & $10^{-6}$ & 168.5 & $25 \times 10^{-6}$ \\
\hline
\end{tabular}

Figure 8. Experimental system of the FBGs strain test system: (a) Picture. (b) Schematic.

(a)

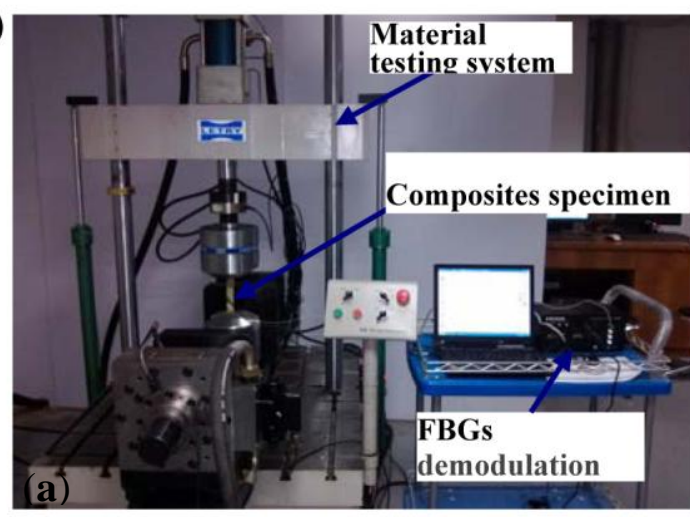

(b)

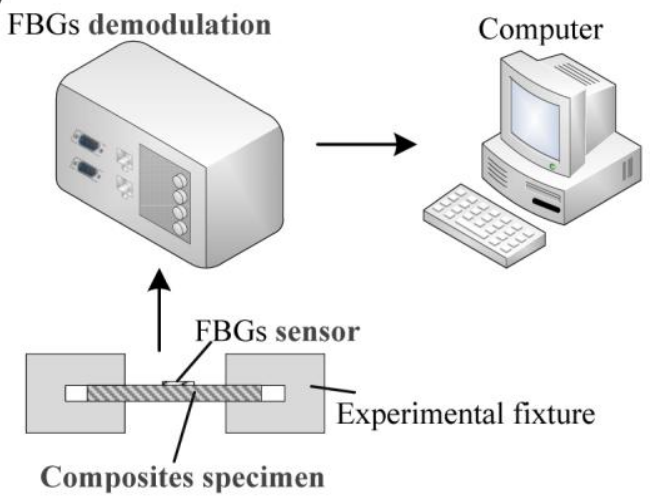

Figure 9. Experimental research on polymer-matrix composites: (a) Strain rate 0.00001/s.

(b) Strain rate $0.01 / \mathrm{s}$.
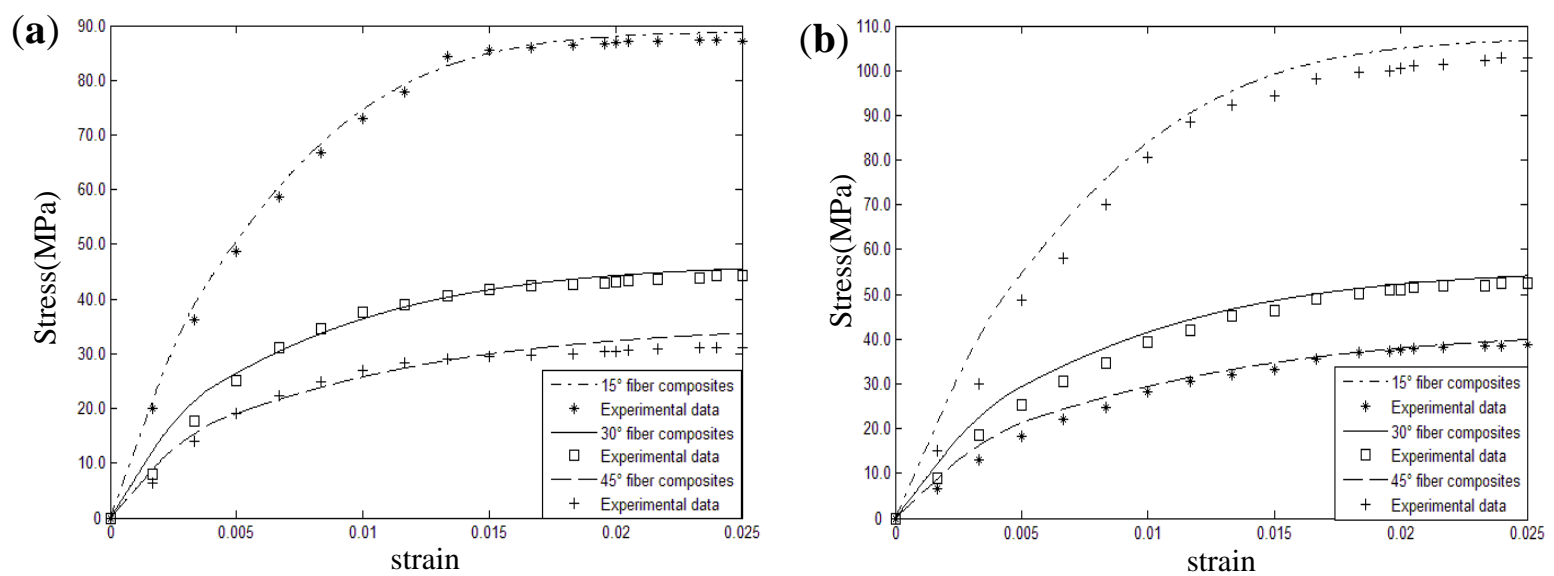

\section{GMC-Based Prediction of Biaxial Yield Response}

Good correlation has been found between the micromechanical model and the experimental data. On this basis, the biaxial yield responses of polymer-matrix composites are studied.

\subsection{Yield Surface Definitions}

With the increase of loading, material deformation is translated from the elastic to the plastic state. This procedure is called yield. A yield surface is defined as the locus of points in a stress or strain space when a specified yield criterion is satisfied. Stress state of materials can be easily discerned by 
the stress yield surface. Generally speaking, the relationship between stress $\sigma_{i j}$, strain $\varepsilon_{i j}$, time $t$ as well as temperture $T$ and yield function $\Phi$ can be written as:

$$
\Phi\left(\sigma_{i j}, \varepsilon_{i j}, t, T\right)=0
$$

Under the condition of ignoring parameters $t$ and $T$, yield function is related to stress and strain of the materials. In the stress space, when the load stress cannot reach the yield surface, the materials can be considered to be in an elastic state. Once stress lies on the yield surface, the materials begin to yield.

Many researchers have studied the yield surface of materials by experimental methods. The results show that the yield surface presents different shapes. Ishikawa [22] indicated that subsequent yield surface presents elliptic shapes without a shape corner and cross effect. However, Kan et al. [23,24] pointed out that a sharp corner in preloading direction and cross effect on the normal preloading direction in condition of complicated loading can be observed. The different results derive from different definition of yield $[25,26]$. At present, three important yield criteria for composites can be expressed as follows [6,27]:

(1) $\sqrt{\dot{\varepsilon}_{i j}^{I} \dot{\varepsilon}_{i j}^{I}}$ is defined as surfaces of constant inelastic strain rate (SCISRs)

(2) $\bar{\sigma}_{i j} \dot{\varepsilon}_{i j}^{I}$ is defined as surfaces of constant inelastic power (SCIPs)

(3) $\varepsilon_{p s}=\sqrt{\frac{2}{3} \varepsilon_{i j}^{I} \varepsilon_{i j}^{I}}$ is defined as surfaces of equivalent plastic strain (SEPSs)

where $\bar{\sigma}_{i j}$ refers to average stress. $\varepsilon_{p s}$ is equivalent plastic strain. $\dot{\varepsilon}_{i j}^{I}$ and $\varepsilon_{i j}^{I}$ indicate inelastic strain rate and inelastic strain of the materials.

In order to build a fiber-reinforced composites yield surface, equivalent plastic strain $\varepsilon_{p s}=0.001$ is used to investigate the biaxial yield response of composites in this paper. For each condition, biaxial yield responses are mapped out by a constant strain ratio, namely, $\varepsilon_{x x} / \varepsilon_{y y}=$ constant or $\varepsilon_{x x} / \varepsilon_{x y}=$ constant.

\subsection{Thermal Residual Stress and Strain Rate Influence on Biaxial Yield Response}

As mentioned in references [28,29], uniaxial inelastic deformation of composites is deeply dependent on thermal residual stress and strain rate. The two parameters' influence the biaxial yield response of polymer-matrix composites is discussed in this section. In these cases, circular fiber-reinforced composites with 0.515 fiber volume fraction are considered. Thermal expansion coefficients of the glass fiber and matrix can be seen in Table 2. The thermal residual stress calculation can be seen in reference [30].

\subsubsection{Thermal Residual Stress Influence on Yield Surface}

For biaxial loading under constant strain rate conditions $\left(\dot{\varepsilon}_{x x}=\dot{\varepsilon}_{y y}=10^{-5}\right.$ or $\dot{\varepsilon}_{x x}=\dot{\varepsilon}_{x y}=10^{-5}$ ), thermal residual stress effects on the yield surface of $15^{\circ}, 30^{\circ}, 45^{\circ}$ fiber-reinforced composites are discussed. For comparison purposes, the yield surface of composites disregarding the thermal stress effect is also shown in the corresponding figures. Figures 10 and 11 show the yield surface in the $\sigma_{x x}-\sigma_{y y}$ and $\sigma_{x x}-\sigma_{x y}$ stress planes, respectively. The temperature drop was assumed to be $150{ }^{\circ} \mathrm{C}$ 
in the simulation examples. Taking Figure 10(b) as an example, it can be seen that the yield surfaces of composites with thermal stress and without thermal stress have two intersections, namely intersection A and B.

The region above the line A-B is defined as region I, while the other one is defined as region II. From the Figures 10 and 11, it can be seen that the biaxial yield response of composites had a characteristic two-region deformation behavior. However, the thermal residual stress effects on the yield surface of composites exhibits opposite variation between the $\sigma_{x x}-\sigma_{y y}$ and $\sigma_{x x}-\sigma_{x y}$ stress planes. In details, the biaxial yield strength of composites in the $\sigma_{x x}-\sigma_{y y}$ stress plane tends to be increased to a certain extent when the thermal residual stress is taken into account in region I, while the thermal residual stress tends to decrease the yield strength in region II. However, it is interesting to mention that the opposite variation can be found in the $\sigma_{x x}-\sigma_{x y}$ stress plane. The yield strength intends to decrease when the thermal residual stress is taken into account in region I. However, thermal residual stress intends to increase the biaxial yield strength when the load is located in region II. Furthermore, comparing with other $\sigma_{x x}-\sigma_{x y}$ stress conditions, the thermal residual stress effects on the biaxial yield response for $15^{\circ}$ fiber-reinforced composites can be ignored.

Figure 10. Thermal residual stress influence on $\sigma_{x x}-\sigma_{y y}$ yield surface of composites: (a) $15^{\circ}$ fiber-reinforced composites; (b) $30^{\circ}$ fiber-reinforced composites; (c) $45^{\circ}$ fiber-reinforced composites.
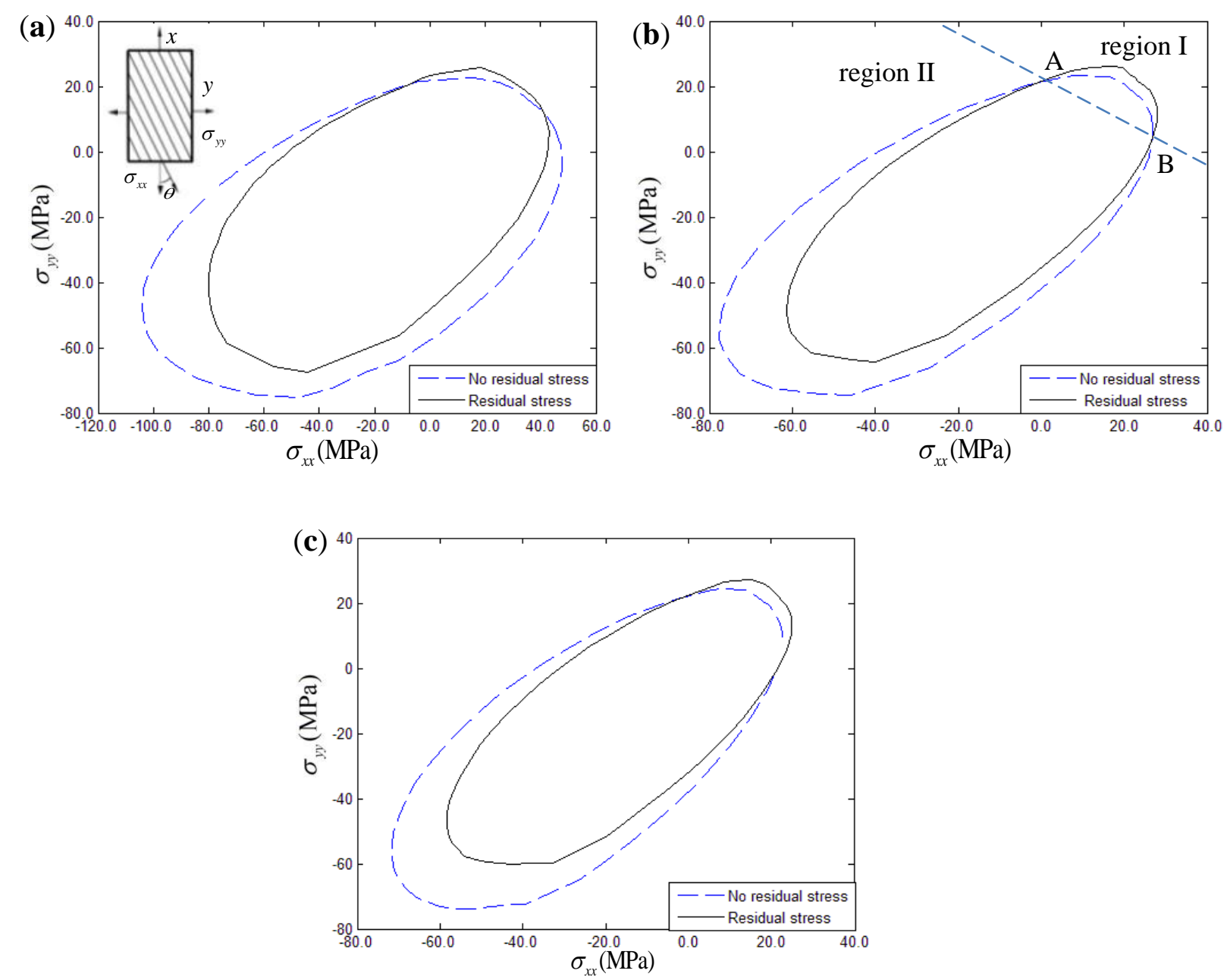
Figure 11. Thermal residual stress influence on the $\sigma_{x x}-\sigma_{x y}$ yield surface of composites:

(a) $15^{\circ}$ fiber-reinforced composites; (b) $30^{\circ}$ fiber-reinforced composites;

(c) $45^{\circ}$ fiber-reinforced composites.
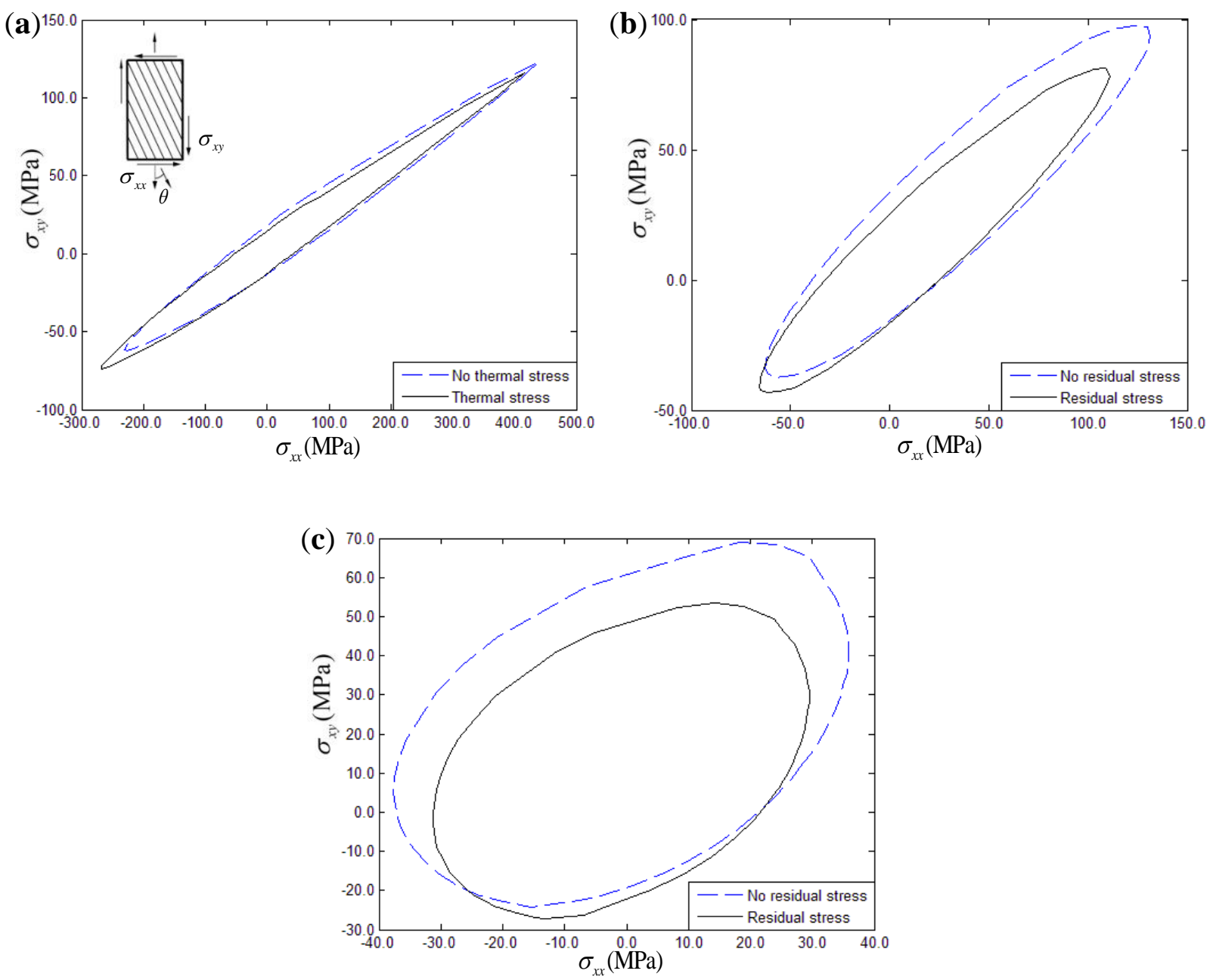

\subsubsection{Strain Rate Influence on Yield Surface}

The effects of a strain rate range of $0.0001 / \mathrm{s}$ to $0.01 / \mathrm{s}$ on the biaxial yield strength of fiber-reinforced composites with thermal residual stress under $\sigma_{x x}-\sigma_{y y}$ and $\sigma_{x x}-\sigma_{x y}$ conditions can be seen in Figures 12 and 13, respectively. Three different fiber off-axis angles $\left(15^{\circ}, 30^{\circ}, 45^{\circ}\right)$ are discussed. From the figures, it can be found that the biaxial yield strength exhibits a significantly rate-dependence in the $\sigma_{x x}-\sigma_{y y}$ and $\sigma_{x x}-\sigma_{x y}$ stress planes similar to the uniaxial loading conditions. Increasing the strain rate will increase the biaxial yield strength of polymer-matrix composites. Furthermore, strain rate effects on the biaxial yield surface can be hardly discerned if the fiber off-axial angle is $15^{\circ}$ in the $\sigma_{x x}-\sigma_{x y}$ stress plane. 
Figure 12. Strain rate influence on $\sigma_{x x}-\sigma_{y y}$ yield surface of composites:

(a) $15^{\circ}$ fiber-reinforced composites; (b) $30^{\circ}$ fiber-reinforced composites; (c) $45^{\circ}$ fiber-reinforced composites.
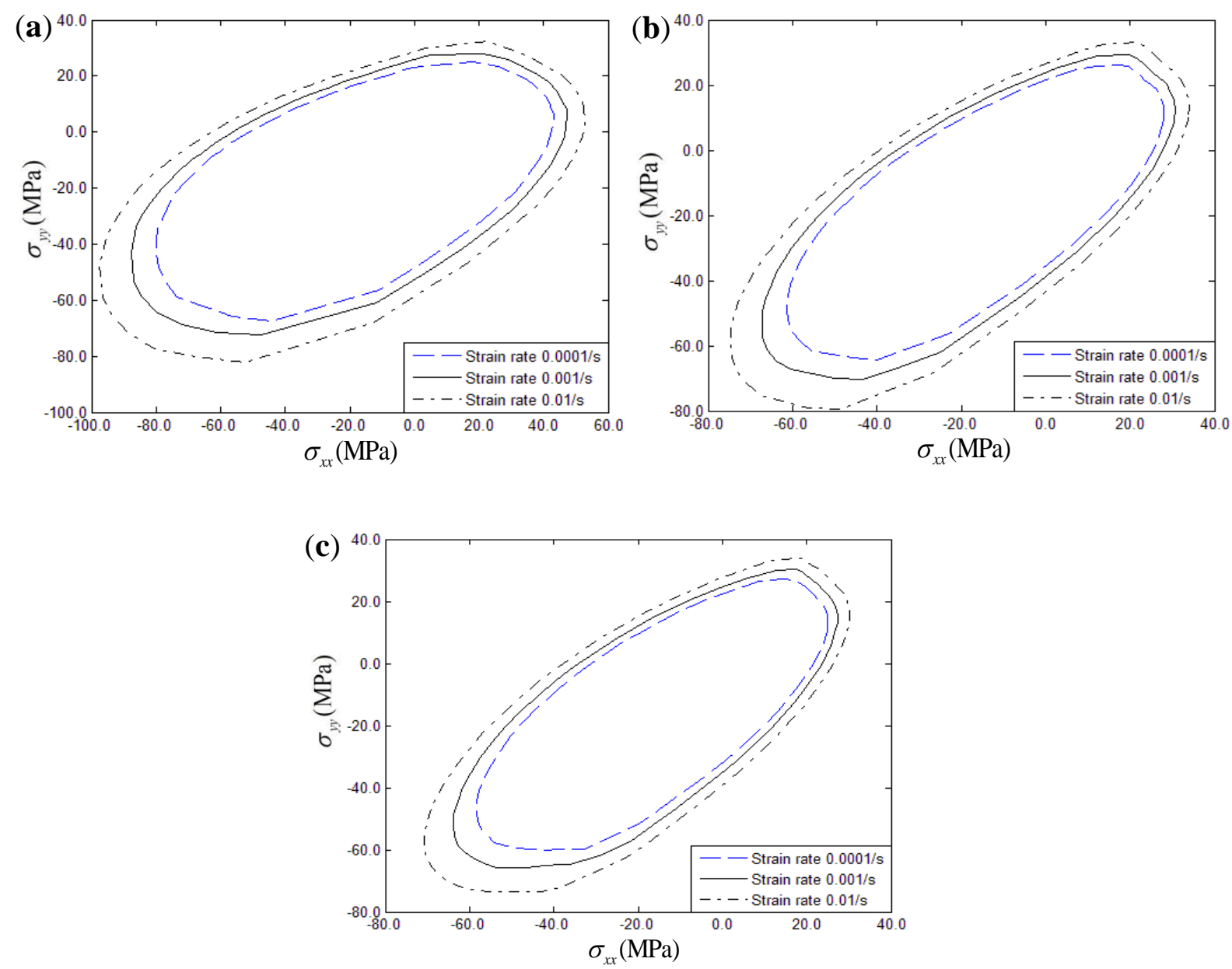

Figure 13. Strain rate influence on $\sigma_{x x}-\sigma_{x y}$ yield surface of composites: (a) $15^{\circ}$ fiber-reinforced composites; (b) $30^{\circ}$ fiber-reinforced composites; (c) $45^{\circ}$ fiber-reinforced composites.
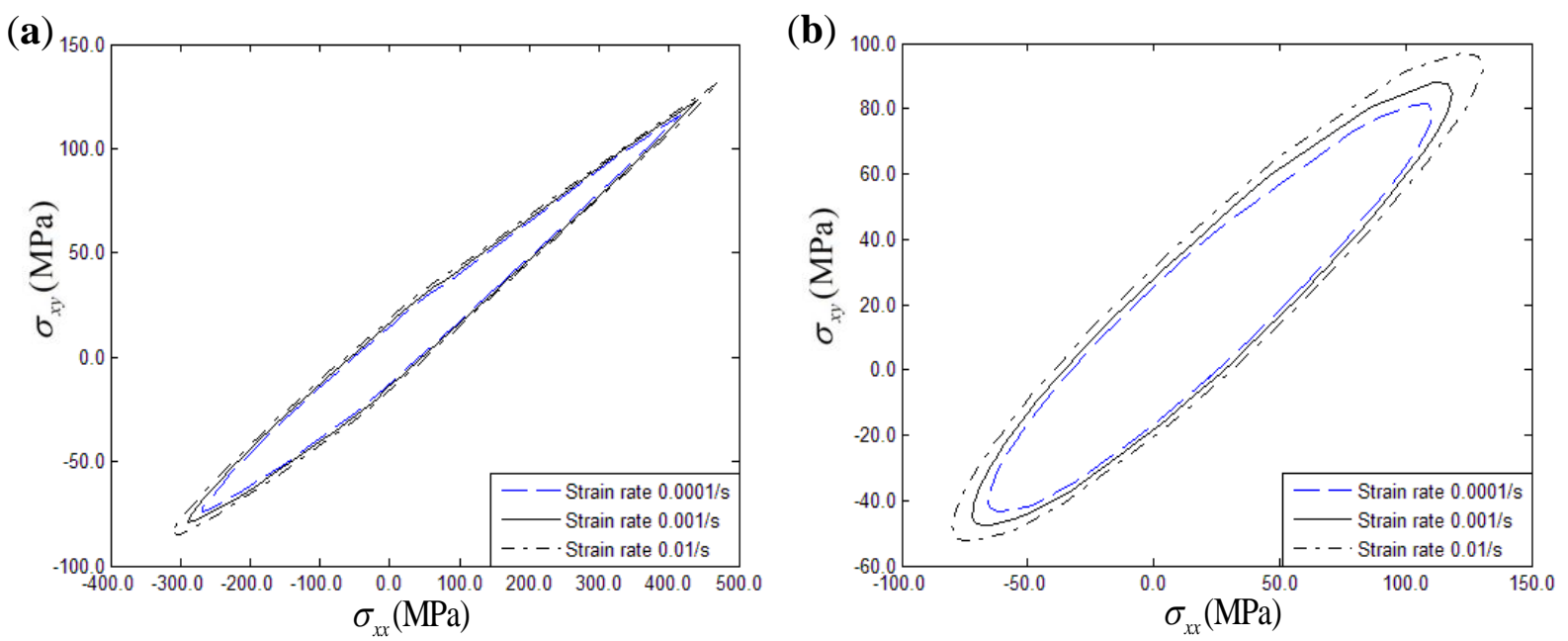
Figure 13. Cont.

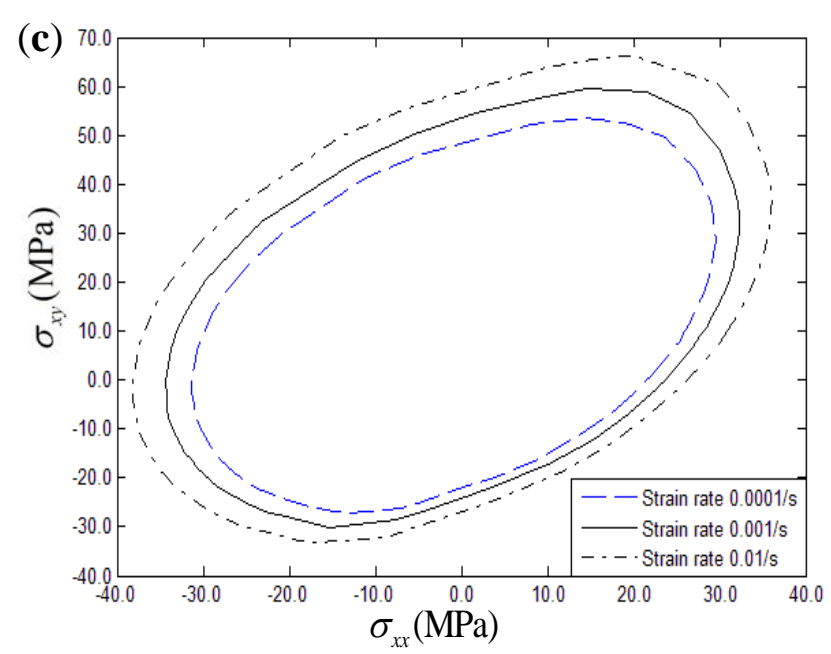

\section{Conclusions}

The Generalized Method of Cells can be used to predict the nonlinear stress-strain of metal-matrix composites, polymer-matrix composites and ceramic-matrix composites through incorporating different viscoplastic models. In this paper, the method has been used to investigate the thermal residual stress and strain rate influence on the biaxial yield responses of polymer-matrix composites with different fiber off-axis angles. In the $\sigma_{x x}-\sigma_{y y}$ stress plane, thermal residual stress tends to increase and decrease the biaxial yield strength of composites in region I and region II, respectively. However, the law for the $\sigma_{x x}-\sigma_{x y}$ stress plane, which is influenced by thermal residual stress, shows the opposite variation. In addition, increasing strain rate tends to increase the yield strength of composites in both the $\sigma_{x x}-\sigma_{y y}$ and $\sigma_{x x}-\sigma_{x y}$ stress planes, which is unrelated to the fiber off-axis angle.

\section{Acknowledgements}

This work was supported by the National Natural Science Foundation of China (No. 51175397), the key project of the National Natural Science Foundation of China (No. 51035007) and the First Aircraft Institute of Aviation Industry Corporation of China (HX0112040106).

\section{References}

1. Azizi, R.; Niordson, C.F.; Brian, N.L. Size-effects on yield surfaces for micro reinforced composites. Int. J. Plasticity 2011, 27, 1817-1832.

2. Tang, T.; Yu, W.B. Asymptotical approach to initial yielding surface and elastoplasticity of heterogeneous materials. Mech. Adv. Mater. Sturct. 2011, 18, 244-254.

3. Moshtaghin, A.F.; Naghdabadi, R.; Asghari, M. Effects of surface residual stress and surface elasticity on the overall yield surfaces of nanoporous materials with cylindrical nanovoids. Mech. Mater. 2012, 51, 74-87.

4. Acton, K.; Graham, L. Meso-scale modeling of plasticity in composites. Comput. Method. Appl. M. 2009, 198, 920-932 
5. Acton, K.; Graham, L. Fitting an anisotropic yield surface using the generalized method of cells. Solid. Mech. Appl. 2009, 168, 27-41.

6. Selmi, A.; Doghri, I.; Adam, L. Micromechanical simulations of biaxial yield, hardening and plastic flow in short glass fiber reinforced polyamide. Int. J. Mech. Sci. 2011, 53, 696-706.

7. Radi, M.; Abdul, A. A self-consistent approach describing the strain induced anisotropy: Case of yield surface evolution. Comp. Mater. Sci. 2012, 54, 356-369.

8. Lissenden, C.J. Experimental investigation of initial and subsequent yield surfaces for laminated metal matrix composites. Int. J. Plasticity 2010, 26, 1606-1628.

9. Lissenden, C.J.; Lei, X. A more comprehensive method for yield locus construction for metallic alloys and composites. Exp. Mech. 2004, 44, 10-20.

10. Ye, J.J.; Chen, X.F.; Zhai, Z.; Li, B.; Duan, Y.G.; He, Z.J. Predicting the elastoplastic response of fiber-reinforced metal matrix composites. Mech. Compos. Mater. 2010, 46, 405-416.

11. Rami, H.A.; Aboudi, J. Nonlinear micromechanical formulation of the high fidelity generalized method of cells. Int. J. Solids Struct. 2009, 46, 2577-2592.

12. Rami, H.A.; Aboudi, J. Formulation of the high-fidelity generalized method of cells with arbitrary cell geometry for refined micromechanics and damage in composites. Int. J. Solids Struct. 2010, 47, 3447-3461.

13. Ho, S.C.M.; Razavi, M.; Nazeri, A.; Song, G.B. FBG sensor for contact level monitoring and prediction of perforation in cardiac ablation. Sensors 2012, 12, 1002-1013.

14. Guo, H.L.; Xiao, G.Z.; Mrad, N.; Yao, J.P. Fiber optic sensors for structural health monitoring of air platforms. Sensors 2011, 11, 3687-3705.

15. Antunes, P.; Travanca, R.; Rodrigues, H.; Melo, J.; Jara, J.; Varum, H.; André, P. Dynamic structural health monitoring of slender structures using optical sensors. Sensors 2012, 12, 6629-6644.

16. Ebrahim, A.F.; Osman, N.A.; Adikan, F.R.M. The use of fiber Bragg grating sensors in biomechanics and rehabilitation applications: The state-of-the-art and ongoing research topics. Sensors 2012, 12, 12890-12926.

17. Leng, J.S.; Asundi, A. Structural health monitoring of smart composite materials by using the extrinsic fabry-perot interferometer and fibre Bragg grating sensors. Sens. Actuators A 2003, 103, $330-340$

18. Luyckx, G.; Voet, E.; Lammens, N.; Degrieck, J. Strain measurements of composite laminates with embedded Fibre Bragg Gratings: Criticism and opportunities for research. Sensors 2011, 11, 384-408.

19. Micron Optics International Corporation Press. The Manual Fiber Bragg Grating Demodulation Instrument (Sm125/Sm130), 1st ed.; The Micron Optics International Corporation Press: Beijing, China, 2010.

20. Yoon, K.J.; Sun, C.T. Characterization of elastic-viscoplastic properties of an AS4/PEEK thermoplastic composite. J. Compos. Mater. 1991, 25, 1277-1296.

21. Bodner, S.R. Unified Plasticity for Engineering Application; Kluwer Academic/Plenum Publishers: New York, NY, USA, 2002; pp. 3-22.

22. Isikawa, H. Subsequent yield surface probed from its current center. Int. J. Plasticity 1997, 13, 533-549. 
23. Khan, A.S.; Pandey, A.; Stoughton, T. Evolution of subsequent yield surfaces and elastic constants with finite plastic deformation. Part I: A very low work hardening aluminum alloy (Al6061-T6511). Int. J. Plasticity 2009, 25, 1611-1625.

24. Khan, A.S.; Pandey, A.; Stoughton, T. Evolution of subsequent yield surfaces and elastic constants with finite plastic deformation. Part II: A very high work hardening aluminum alloy (annealed $1100 \mathrm{Al}$ ). Int. J. Plasticity 2010, 26, 1421-1431.

25. Brenner, R.; Lebensohn, R.A.; Castelnau, O. Elastic anisotropy and yield surface estimates of polycrystals. Int. J. Solids Struct. 2009, 46, 3018-3026.

26. Khan, A.S.; Pandey, A.; Stoughton, T. Evolution of subsequent yield surfaces and elastic constants with finite plastic deformation. Part III: Yield surface in tension-tension stress space (Al 6061-T 6511 and annealed 1100 Al). Int. J. Plasticity 2010, 26, 1432-1441.

27. Lissenden, C.J.; Arnold, S.M. Critique of Macro Flow/Damage Surface Representations for Metal Matrix Composites Using Micromechanics; American Society of Mechanical Engineers: Atlanta, GA, USA, 1996.

28. Tsai, J.L.; Chi Y.K. Investigating thermal residual stress effect on mechanical behaviors of fiber composites with different fiber arrays. Compos. Part B Eng. 2008, 39, 714-721.

29. Raimondo, L.; Iannucci, L.; Robinson, P.; Curtis, P.T. Modelling of strain rate effects on matrix dominated elastic and failure properties of unidirectional fibre-reinforced polymer-matrix composites. Compos. Sci. Tech. 2012, 72, 819-827.

30. Ye, J.J.; Chen, X.F.; Zhai, Z.; Li, B.; Zi, Y.Y.; He, Z.J. Effects of thermal stress and imperfect interfacial bonding on the mechanical behavior of composites subjected to off-axis loading. Mater. Sci. Eng. A Struct. 2010, 527, 7530-7537.

(C) 2013 by the authors; licensee MDPI, Basel, Switzerland. This article is an open access article distributed under the terms and conditions of the Creative Commons Attribution license (http://creativecommons.org/licenses/by/3.0/). 